index ecomunicación | nº 10(3) 2020 | Páginas 129-151

E-ISSN: 2174-1859 | ISSN: 2444-3239 | Depósito Legal: M-19965-2015

Recibido el 14_09_2020 | Aceptado el 30_09_2020 | Publicado el 12_11_2020

\title{
PERIODISMO DIGITAL Y ALTA TECNOLOGÍA: DE LA CONSOLIDACIÓN A LOS RENOVADOS DESAFÍOS
}

\section{DIGITAL JOURNALISM AND HI-TECH: FROM CONSOLIDATION TO RENEWED CHALLENGES}

https://doi.org/10.33732/ixc/10/03Period

\begin{abstract}
Sara Pérez-Seijo
Universidade de Santiago de Compostela s.perez.seijo@usc.es https://orcid.org/0000-0002-5674-1444
\end{abstract}

Beatriz Gutiérrez-Caneda Universidade de Santiago de Compostela beatriz.gutierrez@rai.usc.es https://orcid.org/0000-0002-7191-1886

Xosé López-García Universidade de Santiago de Compostela xose.lopez.garcia@usc.es https://orcid.org/0000-0002-1873-8260

Este artículo está elaborado en el marco del proyecto Cibermedios nativos digitales en España: formatos narrativos y estrategia móvil (RTI2018-093346-B-C33), del Ministerio de Ciencia, Innovación y Universidades, cofinanciado por el Fondo Europeo de Desarrollo Regional (FEDER). Además, la autora Sara Pérez-Seijo es beneficiaria del programa de Formación del Profesorado Universitario (FPU) financiado por el Ministerio de Universidades (Gobierno de España). 
Resumen: El periodismo digital se dirige hacia su tercera década con los deberes de consolidar el campo hecho y con renovados desafíos, que afronta a partir de la experiencia cosechada, el contexto actual pos-COVID-19, el protagonismo que ha ganado la dimensión tecnológica y la necesidad de metodologías sólidas para analizar la complejidad actual. La nueva etapa se construye de la mano de equipos estables de investigación en la práctica totalidad de las universidades españolas con estudios de periodismo, que han avanzado gracias a los estudios pioneros de pequeños grupos encabezados por Ramón Salaverría y Javier Díaz-Noci, hace algo más de veinticinco años, y que ahora, tras ganar posiciones en el contexto internacional, realizan una intensa actividad que aporta conocimiento de forma constante. En este artículo se analiza esta evolución y se presta especial atención, partiendo de una revisión sistematizada de la literatura científica en el campo, a los renovados desafíos que llegan de la mano de la aplicación al campo periodístico de la inteligencia artificial, la realidad virtual, el 5G, los drones y la tecnología blockchain, entre otras altas tecnologías.

Palabras clave: periodismo digital; ciberperiodismo; alta tecnología; inteligencia artificial; periodismo inmersivo; periodismo automatizado.

\begin{abstract}
Digital journalism walks towards its third decade of life with the duties of consolidating the field and with renewed challenges, which it faces based on the gained experience, the post-COVID-19 context, the prominence of the technological dimension and the need for solid methodologies to analyse the current complexity. The new stage is built on solid research teams in almost all the Spanish universities with Journalism studies, which have advanced following pioneering studies of small groups led by Ramón Salaverría and Javier Díaz-Noci, more than twenty-five years ago. Now, after gaining positions in the international context, these teams are carrying out an intense activity that brings constant knowledge. This article analyses this evolution and pays particular attention, based on a Systematic Literature Review in the field of Journalism, to the renewed challenges brought by the application of high technologies - artificial intelligence, virtual reality, 5G, technology blockchain, and so on - to the Journalism.
\end{abstract}

Keywords: Digital Journalism; Online Journalism; Hi-tech; Artificial Intelligence; Immersive Journalism; Automated Journalism. 


\section{Introducción}

Las investigaciones más recientes en el campo de la comunicación digital confirman que la investigación sobre periodismo digital es una disciplina afianzada y en desarrollo, pero que tiene pendientes diversos desafíos metodológicos y temáticos (Salaverría, 2019). El camino recorrido para alcanzar esta meta ha contado con numerosos actores del mundo de la investigación en comunicación de las universidades españolas que han hecho una apuesta por un escenario emergente que se construyó alrededor de la red internet y de las Tecnologías de la Información y la Comunicación (TICs). Desde principios de la última década del siglo XX, hubo aportaciones sobre las nuevas maneras de consumir y producir información por vía telemática (Pérez-Luque y Fernández-Arroyo, 1995; Rodríguez-De-las-Heras, 1991) y es en el año 1996 cuando se publicó la que se podría considerar la primera monografía exclusivamente dedicada al periodismo en línea, titulada El periodismo electrónico. Información y servicios multimedia en la era del ciberespacio, de Armañanzas, Díaz-Noci y Meso-Ayerdi (Masip y Micó-Sanz, 2010), que abrieron una línea de trabajo a la que luego se sumaron numerosas contribuciones.

El acontecimiento más importante que les ha ocurrido a los medios de comunicación entre 1995 y 2005 ha sido el nacimiento de un nuevo y de una nueva forma de hacer periodismo (Salaverría, 2008). Este hecho no solo cambió el escenario comunicativo, al que migraron los medios tradicionales y al que se incorporaron los medios nativos digitales, sino que alimentó la construcción de un ecosistema en el que emergió una especialidad periodística, el periodismo digital o ciberperiodismo, y situó al periodismo ante una nueva etapa marcada por la alargada sombra de una renovada dimensión tecnológica. En el periodismo se mantuvo lo básico, los elementos esenciales, pero los desafíos contribuyeron a que esta técnica de comunicación explorase territorios hasta ese momento desconocidos.

En la segunda década del siglo XXI, al calor de la nueva fase de la construcción de la sociedad red, con la emergencia de la web social, el papel de las audiencias activas y el impulso de la inteligencia artificial, el periodismo ha vivido una auténtica metamorfosis (Vázquez-Herrero, Direito-Rebollal, Silva-Rodríguez y López-García, 2020) que le ha colocado ante nuevos retos en medio de un panorama donde todos los datos apuntan a que no estamos ante un punto de llegada, sino más bien ante un punto de partida a nuevas dimensiones. Los constantes cambios que se producen en el ecosistema comunicativo incentivan viejos y nuevos debates, al tiempo que demandan renovadas metodologías para conocer mejor la complejidad que entraña el periodismo actual y aportar conocimiento que nos permita construir una sociedad mejor informada. 


\subsection{Sobre las miradas necesarias}

El periodismo encara la tercera década del tercer milenio desde un campo científico en el que cada vez se advierte más complejidad, lo que demanda renovados y múltiples enfoques a partir del concepto de campo de Bourdieu (Benson y Neveu, 2005), y en el que la consolidación del periodismo digital, en su diversidad de modelos y con los medios nativos avanzando posiciones en el renovado ecosistema comunicativo, concentra buena parte de las investigaciones. La importancia del periodismo como institución creadora de conocimiento en las sociedades actuales y su influencia en los comportamientos de los ciudadanos en la sociedad red ha contribuido al creciente interés académico por el estudio de la epistemología periodística desde distintos ángulos, con importantes avances en el subcampo de las epistemologías del periodismo digital (Ekström y Westlund, 2019). Es necesario seguir avanzando en estas investigaciones y, al mismo tiempo, ampliar la mirada y enfoque para entender la gran diversidad que se vive actualmente en el campo periodístico (Witschge y Deuze, 2020).

Cuando la desinformación que emerge en la sociedad red está desafiando el papel social y la autoridad del periodismo (Ekström, Lewis y Westlund, 2020), la investigación en periodismo digital muestra, a juzgar por la revisión de la producción científica internacional realizada (Salaverría, 2019) o por los estudios sobre la producción científica en el campo de la comunicación en España en los últimos veinticinco años (Martínez-Nicolás, Saperas y CarrascoCampos, 2019), una amplia y variada producción que confirma la existencia de una masa crítica académica dispuesta a asumir el desafío. Esta nueva etapa se caracteriza por un contexto en el que las tecnologías digitales y la «alta tecnología» tienen un renovado papel, en la medida en que las tecnologías digitales han transformado el ejercicio de influencia social al empoderar a sujetos y otorgarles considerable capital de influencia personal (Casero-Ripollés, 2020), y que sitúan al periodismo digital y a los periodistas en un nuevo escenario, lo que influye en su rol y en las relaciones con otros actores de la esfera pública digital.

Los investigadores en el campo periodístico contemplan el panorama con nuevas miradas, que van desde las que en el inicio de la segunda década de este milenio señalaron la relevancia de un escenario caracterizado por un sistema híbrido de viejos y nuevos medios (Chadwick, 2013), hasta los enfoques más recientes en los que se indica que la industria de las noticias, como se ha organizado tradicionalmente, puede no ser necesaria para que sobreviva el periodismo y para que el trabajo de los periodistas siga siendo relevante para la vida de los ciudadanos (Deuze, 2019). La complejidad del escenario exige respuestas desde el campo periodístico así como desde otros campos científicos, a fin de 
disponer de visiones interdisciplinarias que permitan una mejor comprensión de los distintos elementos que conforman el objeto de estudio.

Desde el ámbito de los estudios periodísticos hay algunas previsiones que a día de hoy parecen certezas, como que el futuro del periodismo es en red (Van der Haak, Parks y Castells, 2012), pero hay muchas dudas acerca de a dónde nos conducirá la transición digital que ahora alimenta la inteligencia artificial (Toural-Bran, Vizoso, Pérez-Seijo, Rodríguez-Castro y Negreira-Rey, 2020), con unos sitios de redes sociales como Facebook y Twitter que se han convertido en una parte clave de las dietas de noticias de los usuarios en línea (Kümpel, 2020), y una variada gama de tecnologías, al tiempo que hay consciencia de la existencia de numerosos desafíos en el futuro. Los propios medios digitales están transformando la producción y distribución de noticias, lo que da lugar a nuevas formas de comprenderlas y conceptualizarlas (Bengtsson y Johansson, 2020).

\subsection{Periodismo de alta tecnología}

Las tecnologías actuales indican el camino al futuro con varias opciones y con unos medios migrantes y otros nativos digitales que experimentan e innovan para conquistar nuevos territorios. En el punto de partida, el que fija el escenario actual, hay muchas iniciativas de periodismo digital que muestran la existencia de vías para asegurar el futuro del periodismo, porque el hardware y el software, si se emplean adecuadamente, permiten formatos innovadores y productos que muestran buen periodismo. En la actualidad sabemos que hay renovados desafíos que llegan de la mano de la aplicación al campo periodístico de la inteligencia artificial, la realidad virtual, el 5G o la tecnología blockchain, entre otras altas tecnologías.

Salaverría (2015) definió por primera vez el periodismo de alta tecnología o periodismo hi-tech como una modalidad periodística que incluye desde noticias escritas por robots o algoritmos e imágenes obtenidas con drones hasta, entre otros, contenidos generados con técnicas de realidad virtual. Desde entonces, otros autores han prestado atención al fenómeno analizando las distintas formas que adopta el periodismo que «contará el futuro» (López-Hidalgo, 2016) - periodismo dron, periodismo inmersivo, periodismo de realidad aumentada, etc. - , así como a los retos y desafíos que, desde el punto de vista laboral, empresarial y de la calidad de la información, representa la aplicación de tecnologías emergentes tanto en la práctica como en el ámbito periodístico (Murcia y Ufarte, 2019).

A partir de estas aproximaciones al fenómeno denominado como periodismo hi-tech o de alta tecnología, se propone una primera definición con el fin 
de contribuir a su correcta identificación y a la comprensión de este fenómeno que, al igual que la tecnología, no deja de ser emergente.

El periodismo de alta tecnología consiste en la aplicación de tecnologías digitales sofisticadas y/o emergentes en distintos ámbitos del periodismo, de tal forma que su uso contribuye en alguna medida (tiempo, coste, análisis, comprensión, impacto, perspectiva, etc.) a la mejora del proceso (por ej., el periodismo automatizado), de la experiencia (por ej., el periodismo inmersivo) o incluso del modelo (por ej., la tecnología blockchain). Dentro de este «aglutinador» periodismo hi-tech se incluyen modalidades o tendencias periodísticas como el periodismo dron, el periodismo inmersivo con vídeo 360 grados y realidad virtual y el periodismo robot o automatizado, así como la producción y aplicaciones periodísticas mediante tecnologías como el blockchain y la realidad aumentada, entre otras.

Esta definición se presenta como una suerte de aproximación conceptual para hacer frente a los desafíos actuales, cuya vocación es abierta - a nuevas tecnologías y herramientas - y de seguir avanzando en su conformación en este escenario de cambio cuando entran con mucha fuerza en el periodismo la inteligencia artificial y el 5G.

\section{Metodología}

Este trabajo se presenta con el objetivo de analizar los estudios recientes que abordan la integración de la alta tecnología en el periodismo, tanto en general - como fenómeno - como en particular - cada alta tecnología por individual一, para así conocer (1) qué investigaciones se han realizado en este campo, (2) qué altas tecnologías son las más estudiadas y (3) cuáles son los retos, desafíos y previsiones de futuro que los investigadores han identificado a raíz de sus estudios.

Para llevar a cabo este trabajo, se realiza un análisis de la producción científica que aborda la alta tecnología en el periodismo a partir de una revisión sistematizada de los artículos sobre el tema y que están indexados en las bases de datos Web of Science (WoS) y Scopus. Esta estrategia, la revisión sistematizada de literatura o SLR por sus siglas en inglés, permite «identificar, evaluar e interpretar los datos disponibles dentro de un periodo de tiempo de un determinado campo de investigación» (Ramírez-Montoya y García-Peñalvo, 2018: 11).

En concreto, en este estudio analizamos el periodo comprendido entre 2019 y agosto de 2020. La decisión de no retroceder más en el tiempo es la reciente publicación de una revisión bibliográfica sobre los primeros 25 años de investigación sobre periodismo digital - desde el nacimiento de los primeros medios web en 1994 hasta 2018- que, si bien se centra en distintas áreas 
de investigación, también ofrece un repaso de los estudios que han prestado atención al uso de distintas tecnologías en el periodismo/con fines periodísticos (Salaverría, 2019).

Para llevar a cabo la revisión, establecimos en primer lugar los recursos de búsqueda que, como hemos mencionado, se corresponden con las bases de datos Scopus y WoS. A continuación determinamos las palabras claves que utilizaríamos en las búsquedas: periodismo de alta tecnología (hi-tech journalism/high technology journalism); tecnologías emergentes AND periodismo (emerging technologies AND journalism); periodismo automatizado, robotizado o algorítmico (automated/robot/algorithmic journalism); periodismo inmersivo (immersive journalism); periodismo dron (drone journalism); blockchain AND periodismo (blockchain AND journalism); 5G AND periodismo (5G AND journalism); periodismo de realidad aumentada (augmented reality journalism).

Por último, definimos los criterios de inclusión y exclusión que utilizaríamos para llevar a cabo la revisión sistematizada: (1) artículos originales sobre el objeto de estudio, de tal forma que se excluyeron editoriales, comentarios, introducciones, reseñas de libros, resúmenes u otro tipo de publicaciones; (2) documentos disponibles en inglés, en español y/o en portugués; y (3) publicación entre 2019 y 2020. Así pues, se han omitido de la revisión todos aquellos trabajos que no cumplían con los criterios de inclusión y cuyo enfoque o aproximación no guardaba relación con el periodismo de alta tecnología o la aplicación de una alta tecnología en particular en el ámbito periodístico.

\section{Resultados}

En este apartado se presentan los resultados más destacados que se han obtenido de la revisión sistematizada de la producción reciente sobre el periodismo de altas tecnologías y la aplicación de determinadas altas tecnologías al periodismo. En total, se extrajeron de las bases de datos WoS y Scopus 99 artículos que fueron publicados entre 2019 y agosto de 2020.

El posterior análisis del contenido de los artículos obtenidos durante la revisión ha permitido identificar un total de cinco tipos de altas tecnologías o «periodismos de alta tecnología» diferentes: periodismo automatizado (también denominado algorítmico, computacional o robot), periodismo inmersivo (artículos que hacen referencia a tecnologías inmersivas como la realidad virtual o los vídeos $360^{\circ}$ ), periodismo dron, la tecnología blockchain y el periodismo de realidad aumentada. Es importante subrayar que, aunque algunos autores clasifican la realidad aumentada como una tecnología inmersiva más, los autores de esta investigación consideran que se debe analizar por separado ya 
que la realidad aumentada no crea una sensación de presencia en el usuario, sino que añade elementos a su realidad.

Tabla 1. Total de artículos encontrado según el tema

\begin{tabular}{|c|c|c|}
\hline Tema & Palabras clave & $\begin{array}{c}N^{\circ} \text { de } \\
\text { artículos }\end{array}$ \\
\hline $\begin{array}{l}\text { Periodismo de } \\
\text { alta tecnología }\end{array}$ & $\begin{array}{l}\text { hi-tech journalism; high } \\
\text { technology journalism; } \\
\text { emerging technologies } \\
\text { AND journalism }\end{array}$ & 7 \\
\hline $\begin{array}{l}\text { Periodismo } \\
\text { automatizado }\end{array}$ & $\begin{array}{l}\text { automated journalism; } \\
\text { robot journalism; } \\
\text { algorithmic journalism }\end{array}$ & 50 \\
\hline $\begin{array}{l}\text { Periodismo } \\
\text { inmersivo }\end{array}$ & immersive journalism & 30 \\
\hline Periodismo dron & drone journalism & 9 \\
\hline Blockchain & $\begin{array}{l}\text { blockchain AND } \\
\text { journalism }\end{array}$ & 2 \\
\hline $5 G$ & $5 G$ AND journalism & 0 \\
\hline $\begin{array}{l}\text { Periodismo de } \\
\text { realidad } \\
\text { aumentada }\end{array}$ & $\begin{array}{l}\text { augmented reality } \\
\text { journalism }\end{array}$ & 1 \\
\hline & Total & 99 \\
\hline
\end{tabular}

Fuente: elaboración propia.

El periodismo automatizado ha sido el tema más estudiado en la producción científica publicada durante el periodo analizado. De hecho, es abordado en el 51\% de los artículos examinados. En segundo lugar se encuentra el periodismo inmersivo, tendencia que analizan el 30\% de los artículos extraídos durante la revisión. El resto de altas tecnologías han sido notablemente menos investigadas, siendo las que menos la realidad aumentada (1\%), el blockchain (2\%) y el periodismo dron (9\%). De hecho, ni siquiera el periodismo de alta tecnología como fenómeno emergente y general ha copado gran atención en los estudios periodísticos recientes (7\%).

Por otra parte, al analizar la procedencia de los autores que firman los 99 artículos analizados en este trabajo, observamos que proceden de universidades o, en su defecto, organizaciones, de un total de 20 países distintos. Si bien 
se aprecia diversidad en lo relativo a los territorios, destaca que son España y, muy de cerca, Estados Unidos los países con mayor presencia en el corpus de artículos revisados sobre periodismo(s) de alta tecnología.

Tabla 2. Procedencia de los firmantes, centros de adscripción por país y volumen de artículos por territorio

\begin{tabular}{|l|r|r|}
\hline \multicolumn{1}{|c|}{ País } & $\begin{array}{c}\text { No centros } \\
\text { adscripción }\end{array}$ & $\begin{array}{c}\text { No de } \\
\text { artículos }\end{array}$ \\
\hline Alemania & 3 & 3 \\
\hline Austria & 1 & 1 \\
\hline Australia & 4 & 4 \\
\hline Bélgica & 1 & 2 \\
\hline Brasil & 5 & 4 \\
\hline Corea del Sur & 4 & 2 \\
\hline Emiratos Árabes & 1 & 1 \\
\hline España & 19 & 28 \\
\hline Estados Unidos & 20 & 21 \\
\hline Finlandia & 4 & 4 \\
\hline Francia & 2 & 2 \\
\hline India & 2 & 2 \\
\hline Noruega & 2 & 2 \\
\hline Países Bajos & 3 & 7 \\
\hline Portugal & 3 & 2 \\
\hline Qatar & 1 & 1 \\
\hline Reino Unido & 8 & 9 \\
\hline República Checa & 2 & 1 \\
\hline Singapur & 2 & 4 \\
\hline Suiza & 1 & 1 \\
\hline
\end{tabular}

Fuente: elaboración propia.

En lo relativo a las revistas en las que han sido publicados los artículos que abordan el periodismo hi-tech o alguna alta tecnología en particular, se han encontrado un total de 41 cabeceras distintas. Con todo, se observa cómo algunas revistas han publicado un volumen notablemente mayor de estudios sobre el tema. A este respecto, destacan Digital Journalism, con 19 textos, y Media and Communication, con 13. En la tabla 3 se recogen solo las revistas que han publicado más de dos artículos durante el periodo analizado. 
Tabla 3. Revistas que mayor volumen de contenidos han publicado

\begin{tabular}{|l|r|}
\hline \multicolumn{1}{|c|}{ Revista } & $\begin{array}{c}\text { No de } \\
\text { artículos }\end{array}$ \\
\hline Digital Journalism & 19 \\
\hline Media and Communication & 13 \\
\hline Journalism Practice & 6 \\
\hline Revista Latina de Comunicación Social & 5 \\
\hline DOXA COMUNICACIÓN & 5 \\
\hline Journalism Studies & 5 \\
\hline El Profesional de la Información & 3 \\
\hline Journalism & 3 \\
\hline NEW MEDIA \& SOCIETY & 3 \\
\hline Journalism and MasS Communication Quarterly & 3 \\
\hline
\end{tabular}

Fuente: elaboración propia.

\subsection{Periodismo de alta tecnología y tecnologías emergentes}

En este apartado se enmarcan aquellos estudios periodísticos que abordan la alta tecnología y/o las tecnologías emergentes. Si bien la cifra de resultados es relativamente baja, esto se explica en parte por la tendencia de los investigadores a estudiar y analizar tecnologías en particular (inteligencia artificial, realidad virtual, etc.), de tal forma que los trabajos sobre el fenómeno en general resultan menos frecuentes.

Con todo, en los artículos identificados es posible distinguir diferentes enfoques y/o líneas de investigación. Por un lado, se encuentran textos relacionados con la formación de los futuros periodistas en los entornos universitarios. En España, por ejemplo, se han revisado los planes de estudio de los grados de Periodismo y/o Comunicación con el fin de identificar hasta qué punto se forma a los estudiantes en materia de tecnologías emergentes y disruptivas como la inteligencia artificial, la realidad virtual o, entre otras, los drones (Sierra, Liberal y Mañas-Viniegra, 2020; Ufarte, Calvo y Murcia, 2020). Ambos trabajos arrojan conclusiones similares al apuntar que esta oferta formativa no solo es incipiente sino que, además, su incorporación en los programas de las asignaturas es lenta. Otros autores van más allá y proponen un nuevo método de enseñanza-aprendizaje de tecnologías emergentes en el periodismo basado en el sketching, que implicaría dibujo y esbozo (Doherty y Worthy, 2020). Una técnica pensada para estimular el pensamiento creativo sobre la evolución de la práctica periodística al abrigo de los últimos avances tecnológicos, así como para ayudar a los estudiantes a desarrollar aptitudes empresariales.

Por otro lado, se han identificado también trabajos centrados, entre otros, en aspectos como la evolución de la dinámica de poder entre la industria 
de los medios de comunicación y la industria tecnológica (Wang, 2020); o en la forma en que las últimas tecnologías han dado paso a nuevas formas de participación pública y novedosas interfaces de usuario y contenidos cuyo impacto se ha traducido, además, en cambios en las prácticas profesionales (Chua y Westlund, 2019).

\subsection{Periodismo automatizado}

La integración de algoritmos y de la inteligencia artificial en las redacciones periodísticas ha dado paso a una reformulación de ciertas prácticas y tareas que, hasta su llegada, eran desempeñadas por humanos. Si bien Carlson (2015: 417) definió este periodismo automatizado como «procesos algorítmicos que convierten datos en textos narrativos informativos con intervención humana limitada o nula más allá de la programación inicial», en el presente también es posible automatizar tareas de los procesos de recopilación, selección, edición y distribución de informaciones o noticias (Wu, Tandoc y Salmon, 2019a). Esta posibilidad, que se ha visto condicionada por el avance y desarrollo tecnológico, permite extender la noción de periodismo automatizado al conjunto de procesos que han sido automatizados en las redacciones y que, por tanto, no requieren intervención humana (Wu, Tandoc y Salmon, 2019b).

El también denominado periodismo algorítmico (Dörr, 2016) o periodismo robotizado (Lemelshtrich, 2018) ha sido ampliamente estudiado desde comienzos de la segunda década del siglo XXI. Pero lejos de relegarse a una línea de investigación pasada, constituye el enfoque temático del que más textos se han obtenido durante este estudio. En concreto, se han podido identificar un total de 50 textos que han sido publicados entre enero de 2019 y agosto de 2020.

Los estudios encontrados abordan cuestiones relativas, entre otras, a los cambios y transformaciones que la automatización de funciones ha producido en las redacciones en particular ( $\mathrm{Wu}$, Tandoc y Salmon, 2019a) y en el periodismo en general (Wu, Tandoc y Salmon, 2019b); a la percepción de los usuarios de la credibilidad, calidad y/u objetividad de las noticias automatizadas (Graefe y Bohlken, 2020; Liu y Wei, 2019; Tandoc, Yao y Wu, 2020; Waddell, 2019a, 2019b); a la calidad de las piezas redactadas por una inteligencia artificial (Ufarte y Manfredi, 2019); a la responsabilidad jurídica del contenido cuando este ha sido escrito por un bot o algoritmo (Lewis, Sanders y Carmody, 2019); a la automatización en las coberturas deportivas (Kunert, 2020; Rojas, 2019; Rojas y Toural, 2019); al impacto del uso de bots en la relación del medio con la audiencia (Ford y Hutchinson, 2019; Jones y Jones, 2019); al periodismo 
estructurado (Caswell, 2019); o a los retos y riesgos de la personalización de contenidos (Kunert y Thurman, 2019).

\subsection{Periodismo inmersivo}

De la Peña et al. (2010: 291) definieron por primera vez el periodismo inmersivo como «la producción de noticias de forma que la gente pueda obtener una experiencia en primera persona de los eventos o situaciones descritas en los relatos informativos». Si bien no fue hasta finales de 2015 y principios de 2016 cuando saltó al panorama periodístico global como una nueva forma de producir contenido no ficción y ofrecer una experiencia de usuario distinta, hoy es uno de los fenómenos emergentes más destacados del nuevo siglo. Desde entonces, la academia ha estado estudiando y analizando las características narrativas de los contenidos, la emergencia de nuevos géneros periodísticos inmersivos y la evolución de la producción a lo largo de los años.

Sin embargo, los estudios revisados publicados en 2019 y 2020 revelan nuevas vías de estudio entre las que destacan, por ejemplo, las investigaciones de corte experimental sobre el impacto de los vídeos periodísticos en 360 grados en la actitud y comportamiento de los usuarios (Barreda-Ángeles, AleixGuillaume y Pereda-Baños, 2020a, 2020b; Bujić, Salminen, Macey y Hamari, 2020; Kang, O’Brien, Villarreal, Lee y Mahood, 2019; Ma, 2019; Mañas-Viniegra, Veloso y Sierra-Sánchez, 2020; Steinfeld, 2020; Van Damme, All, De Marez y Van Leuven, 2019; Vettehen, Wiltink, Huiskamp, Shaap y Ketelaar, 2019); los análisis sobre los retos éticos que plantea esta nueva modalidad periodística (Aitamurto, 2019) y el rol de la audiencia en el diseño de pautas específicas (Sánchez Laws y Utne, 2019); o las revisiones críticas sobre la capacidad de empatía que numerosos autores atribuyeron al periodismo inmersivo durante el boom inicial (Hassan, 2020). En el marco del estudio de la experiencia de usuario, destaca un estudio de Nielsen y Sheets (2019) sobre las gratificaciones que los receptores obtienen cuando consumen contenido inmersivo. Los autores identifican un total de seis: inmersión, transportación, emoción, empatía, información y control.

Además, se encuentran también otras publicaciones de corte más analítico que estudian contenidos que abordan temáticas sociales (Benítez, Herrera y Benítez, 2019) como los derechos humanos (Kukkakorpi y Pantti, 2020; Palmer, 2020); que ponen en el punto de mira las debilidades y los problemas relativos a la usabilidad de las secciones y apps móviles de realidad virtual de los principales medios de comunicación (Gutiérrez-Caneda, Pérez-Seijo y LópezGarcía, 2020); y que, en un afán por entender y definir mejor este fenómeno emergente, proponen modelos conceptuales (De Bruin, De Haan, Kruikemeirer, 
Lecheler y Goutier, 2020), narrativos (Caerols, Sidorenko y Garrido, 2020) y comunicativos (Paíno y Rodríguez, 2019).

\subsection{Periodismo dron}

Los drones comenzaron a usarse con fines periodísticos a mediados de la década de los 2000, si bien no sería hasta mediados de la segunda década del siglo XXI cuando su empleo se generalizaría en todo el mundo como una alternativa más asequible y segura frente al helicóptero. Estos vehículos aéreos no tripulados (UAVs, siglas en inglés) forman parte del conjunto de altas tecnologías emergentes que están redefiniendo el periodismo y la forma de practicarlo. Al igual que el periodismo inmersivo, el llamado periodismo dron permite ofrecer al usuario nuevas perspectivas e incluso una potencial mayor inmersión en la imagen (Pavlik, 2020), de ahí que sus beneficios hayan captado la atención tanto de grandes medios de carácter nacional como de empresas más pequeñas de enfoque local o regional (Ferguson y Greer, 2019).

Si bien no ha sido un área tan estudiada como otras en este artículo analizadas, destaca que el mayor volumen de estudios incluidos en esta revisión se concentra en 2020. Todos estos han sido publicados en un número temático titulado Journalism from Above: Drones, the Media, and the Transformation of Journalistic Practice publicado por la revista Media and Communication. Estos trabajos aportan conclusiones centradas en el impacto de esta alta tecnología en la práctica periodística. Así, encontramos estudios que indican, por ejemplo, que en el periodismo dron el control es «dual», compartido por un operador y un periodista que deben trabajar e interactuar conjuntamente para dar forma a una pieza visual concreta (Adams, 2020); que el uso de imágenes capturadas con este vehículo aéreo no tripulado contribuye en mayor medida a activar la emoción y la atención del receptor (Mañas, García y Martín, 2020); o que nace en respuesta a la crisis institucional del periodismo actual para asistir al rol normativo de la práctica periodística (Hamilton, 2020).

También se han encontrado estudios de caso que abordan la situación de esta práctica en territorios como Finlandia o España. Uskali, Manninen, Ikonen y Hokkanen (2020), por ejemplo, han analizado la situación en Finlandia y han concluido que el periodismo dron está ampliamente extendido en las redacciones del país, a la par que identifican dos tipos de estrategias en este contexto: la adquisición de un dron para capturar material aéreo o la compra de imágenes por encargo a freenlancers; si bien han reparado en que los medios que más imágenes aéreas utilizan son aquellos que disponen de su propio vehículo aéreo no tripulado. Por otra parte, el estudio sobre el caso español concluye que, en comparación con otros países, la normativa de drones en España es muy 
restrictiva, de tal forma que, además, condiciona e impide el desarrollo del periodismo dron (Gallardo-Camacho y Breijo, 2020).

No obstante, otros autores se han centrado en los retos que suscita esta forma emergente de producir contenido visual. Adams (2019), por ejemplo, sugiere que el uso de imágenes aéreas pone en riesgo el periodismo de calidad en la medida en que se utilizan para proporcionar contexto y no nueva información, si bien con frecuencia se presentan como el material más importante. Mientras que Duncan y Culver (2020) han analizado el grado de aceptación y percepción ética de esta modalidad por parte de la audiencia.

\subsection{Otras altas tecnologías}

Al margen de las altas tecnologías antes comentadas, encontramos otras que, si bien no han sido tan estudiadas entre 2019 y 2020 , igualmente introducen importantes cambios en los medios de comunicación en particular y en el periodismo digital en general. Así, por ejemplo, la tecnología blockhain no ha gozado de gran acogida en las investigaciones publicadas durante el periodo analizado, aunque se presenta como potencial futura línea de estudio. Los textos encontrados al respecto aportan conclusiones centradas en el impacto del modelo blockchain en la publicidad gracias a atributos como, entre otros, la seguridad y la transparencia (Cádima, 2019) o en la implementación de un modelo periodístico basado en esta tecnología, que permitiría establecer una red descentralizada (Kim y Yoon, 2019).

A diferencia de lo que ocurre en el caso de la realidad virtual, apenas se hallan textos que aborden el uso de la realidad aumentada con fines periodísticos. En concreto, tan solo encontramos una referencia que analiza el impacto de esta tecnología como herramienta informativa en los telediarios de la televisión privada Antena 3, pionera en España. Los autores concluyen que su empleo se destina a contenidos informativos a los que se les da mayor valor en la escaleta - como noticias de sociedad, política o internacional- y que, por tanto, tienen una mayor duración en pantalla (Azkunaga, Gaztaka y Eguskiza, 2019). Finalmente, no ha sido posible identificar ninguna referencia sobre la tecnología $5 \mathrm{G}$ y su impacto en el periodismo.

\section{Conclusiones}

La revisión de la producción científica sobre periodismo(s) de alta tecnología publicada entre 2019 y 2020 revela que España y, seguidamente, Estados Unidos encabezan los estudios periodísticos sobre el tema. Son los dos países de los que mayor número de investigadores han firmado las publicaciones más recientes. Además, destaca que son también dos las revistas académicas en las 
que más artículos se han publicado durante el mencionado periodo: Digital Journalism y Media and Communication, esta última a razón del lanzamiento de un monográfico sobre el periodismo dron.

Este trabajo, que parte de una revisión sistematizada de la literatura científica sobre la alta tecnología en el periodismo, ha permitido constatar el impacto de los últimos avances y desarrollos tecnológicos en la industria de los medios y en las prácticas periodísticas. La integración de la alta tecnología en el periodismo digital responde a dos escenarios: por un lado, a la carrera de la innovación emprendida en todo el mundo en un afán por lograr la diferenciación en la masa de medios y, por otro, a una estrategia de engagement concebida no solo para reconectar con los usuarios sino también para atraer nuevas audiencias, sobre todo las más jóvenes. Asimismo, gracias a este estudio, se ha observado que el peso real de la alta tecnología en la reconfiguración del periodismo digital es también alto, pues es la que moldea y da consistencia a los nuevos fenómenos periodísticos que, a su vez, dan paso a nuevas prácticas, perfiles profesionales, modelos, formatos e, incluso, formas de storytelling, entre otros aspectos.

En este sentido, la academia nacional e internacional ha centrado su interés en analizar cómo las altas tecnologías redefinen el periodismo digital al filo de sus treinta años de vida, al mismo tiempo que algoritmos y periodistas aprenden a convivir en las redacciones. Pero, sin duda, han sido el periodismo automatizado (bots, algoritmos, inteligencia artificial, big data) y a continuación el periodismo inmersivo las modalidades que más han acaparado la atención de los investigadores en comunicación. Superada la fase de ensayo y error, de pruebas y primeras experiencias, estos «periodismos» progresan hacia una consolidación que la academia advierte repleta de desafíos. Es por ello que parte de los estudios publicados en 2019 y 2020 se han centrado, sobre todo, en analizar la percepción de los usuarios, el impacto en la credibilidad, la introducción de sesgos, los debates éticos y las implicaciones legales.

Si bien el blockhain, la tecnología $5 \mathrm{G}$ y la realidad aumentada no han gozado del mismo volumen de estudios, se pronostica que tendrán una mayor acogida en la investigación académica del futuro a corto-medio plazo, en consonancia con los últimos avances y la introducción de mejoras.

El periodismo digital entra en su tercera década con los deberes de consolidar los fenómenos emergentes de los últimos años y con renovados desafíos que debe afrontar a partir de la experiencia cosechada, el protagonismo que ha adquirido la dimensión tecnológica — sobre todo la de la alta tecnología o hitech-, la necesidad de metodologías sólidas para analizar la complejidad actual y el contexto pos-COVID-19. Retos que, de acuerdo con las investigaciones 
analizadas en este estudio y publicadas entre 2019 y 2020, pasarían por los conflictos que comprometen la ética profesional del periodista en particular y del periodismo en general; las cuestiones legales que dificultan la atribución de responsabilidades y de los derechos de autor; la percepción de los usuarios de la credibilidad y objetividad informativa cuando los contenidos son fruto de una mediación tecnológica - periodismo automatizado- o han eliminado al periodista de la noticia - periodismo dron, periodismo inmersivo-; el rol del profesional de la información cuando una máquina sustituye su papel como mediador; la aproximación hacia un periodismo de corte más emocional y en cierta medida subjetivo y que puede abrir la puerta al sesgo; o, incluso, el elevado coste económico que la introducción de la más alta tecnología actual representa para las redacciones más modestas y con menor capacidad y recursos.

La alta tecnología dibuja un futuro prometedor, repleto de nuevas posibilidades en las que las apuestas de mejora se dirigen hacia los modelos, procesos y experiencias. Un periodismo digital que permitirá ofrecer nuevas perspectivas en las noticias, una mayor inmersión en la realidad informativa, abaratar ciertas tareas y prácticas, novedosas formas de storytelling que han ido tomando forma en los últimos años de la segunda década del tercer milenio, más precisión a partir del análisis de ingentes conjuntos de datos y agilidad en los procesos de filtrado y publicación gracias a la automatización de ciertos procesos. Empero, tras la consolidación de los nuevos formatos, prácticas, modelos, procesos y hasta fenómenos se vislumbran una serie de renovados desafíos a los que el periodismo digital y los profesionales de la información del presente han de hacer frente para garantizar que el periodismo, ante todo, sigue siendo informativo más allá de la influencia tecnológica.

En cuanto a las limitaciones del estudio, cabe señalar que pese a que son múltiples las tecnologías emergentes que se incorporan al periodismo, la ausencia de una etiqueta común a la hora de referirse a ellas dificulta la tarea de identificar los estudios sobre el tema. En el presente artículo hemos partido de la etiqueta «alta tecnología» y la hemos definido en un afán por identificar los últimos trabajos del área. No obstante, habrán quedado fuera aquellos que, si bien han centrado su interés en el conjunto de estas altas tecnologías, se han referido a estas con otros conceptos. Además, otra de las limitaciones ha sido el idioma. Hemos incluido aquellos trabajos disponibles en inglés, español y/o portugués, mientras que nos hemos visto obligados a prescindir de aquellos redactados en otras lenguas foráneas. También el periodo de análisis (enero 2019 - agosto 2020) representa una limitación pero, tal y como se ha mencionado en el apartado relativo al método, los autores tenían constancia de una revisión bibliográfica previa en la que ya se ofrecía un repaso de los estudios centrados 
en el uso de distintas nuevas tecnologías en el periodismo (Salaverría, 2019), de ahí que se haya seleccionado el inmediato periodo posterior.

\section{Referencias}

ADAMS, C. (2019). Tinker, Tailor, Soldier, Thief: An Investigation into the Role of Drones in Journalism. Digital Journalism, 7(5), 658-677.

https: / / doi.org/10.1080/21670811.2018.1533789

ADAMS, C. (2020). Dual control: Investigating the role of drone (UAV) operators in TV and online journalism. Media and Communication, 8(3), 93100. http://dx.doi.org/10.17645/mac.v8i3.2980

AitamurTo, T. (2019). Normative paradoxes in 360 journalism: Contested accuracy and objectivity. New media \& society, 21(1), 3-19. https://doi.org/10.1177/1461444818785153

ARMAÑANZAS, E.; DÍAZ-NOCI, J. y MESO-AYERDI, K. (1996). El periodismo electrónico. Información y servicios multimedia en la era del ciberespacio. Ariel.

AZKUNAGA, L.; GAZTAKA, I. y EGUSKIZA, L. (2019). Nuevas narrativas en televisión: La Realidad Aumentada en los telediarios de Antena 3. Revista de Comunicación, 18(2), 25-50. http: / /dx.doi.org/10.26441/rc18.2-2019-a2

BarredA-Ángeles, M.; AleiX-Guillaume, S. y PEREdA-Baños, A. (2020a). An «Empathy Machine» or a «Just-for-the-Fun-of-It» Machine? Effects of Immersion in Nonfiction 360-Video Stories on Empathy and Enjoyment. Cyberpsychology, Behavior, and Social Networking, 23(10), 683-688. https://doi.org/10.1089/cyber.2019.0665

BarredA-Ángeles, M.; AleiX-Guillaume, S. y PEREdA-Baños, A. (2020b). Virtual reality storytelling as a double-edged sword: Immersive presentation of nonfiction $360^{\circ}$-video is associated with impaired cognitive information processing. Communication Monographs, 1-20. https: / / doi.org/10.1080/03637751.2020.1803496

BEnGTSSON, S. y JOHANSSON, S. (2020). A phenomenology of news: Understanding news in digital culture. Journalism. https://doi.org/10.1177/1464884919901194

BENÍTEZ, M. J.; HERRERA, S. y BENÍTEZ, E. (2019). Analysis of the immersive social content feature in the Spanish news media. Revista Latina de Comunicación Social, (74), 1655-1679. https://doi.org/10.4185/RLCS-2019-1403en BENSON, R. y NEVEU, E. (2005). Bourdieu and the Journalistic Field. Polity Press. BujIĆ, M.; SALMINEN, M.; MACEY, J. y HAMARI, J. (2020). «Empathy machine»: how virtual reality affects human rights attitudes. Internet Research, 30(5), 1407-1425. https://doi.org/10.1108/INTR-07-2019-0306 
CÁDIMA, F. (2019). A Publicidade face aos novos contextos da era Digital: privacidade, transparência e disrupção. Media \& Jornalismo, 19(34), 35-46. https://doi.org/10.14195/2183-5462_34_3

Caerols, R. C.; Sidorenko, P. S. y Garrido, P. G. (2020). Towards a model of narrative in immersive journalism. Revista Latina de Comunicación Social, (75), 341-364. https: / / www.doi.org/10.4185/RLCS-2020-1430

CARLSON, M. (2015). The robotic reporter: Automated journalism and the redefinition of labor, compositional forms, and journalistic authority. Digital journalism, 3(3), 416-431. https://doi.org/10.1080/21670811.2014.976412

CASERO-RIPOLlÉS, A. (2020). Political influencers in the digital public sphere. Communication \& Society, 33(2), 171-173.

https://doi.org/10.15581/003.33.2.171-173

CASWELL, D. (2019). Structured journalism and the semantic units of news. Digital Journalism, 7(8), 1134-1156.

https://doi.org/10.1080/21670811.2019.1651665

Chadwick, A. (2013). The Hybrid Media System: Politics and Power. Oxford University Press. https://doi.org/10.1111/padm.12108

ChuA, S. y WesTLund, O. (2019). Audience-centric engagement, collaboration culture and platform counterbalancing: A longitudinal study of ongoing sensemaking of emerging technologies. Media and Communication, 7(1), 153-165. https://doi.org/10.17645/mac.v7i1.1760

DE Bruin, K.; DE HAAN, Y.; KRUIKEMEIER, S.; LECHELER, S. y GouTIER, N. (2020). A first-person promise? A content-analysis of immersive journalistic productions. Journalism. https://doi.org/10.1177/1464884920922006

DE la PEÑA, N.; WeIL, P.; LlOBERA, J.; Giannopoulos, E.; PoMÉS, A.; SPANlanG, B.; FRIEDMAN, D.; SÁNCHEZ-VIves, M. V. y SLATER, M. (2010). Immersive journalism: immersive virtual reality for the first-person experience of news. Presence: Teleoperators and virtual environments, 19(4), 291-301. https://doi.org/10.1162/PRES_a_00005

DEUZE, M. (2019). What Journalism Is (Not). Social Media + Society, 5(3). https://doi.org/10.1177/2056305119857202

Doherty, S. y WorThY, P. (2020). Sketching: A Method for Imagining Journalistic Tech. Digital Journalism, 1-18. https://doi.org/10.1080/21670811.2020.1764373

DöRR, K. N. (2016) Mapping the field of Algorithmic Journalism. Digital Journalism, 4(6), 700722. https: / /doi.org/10.1080/21670811.2015.1096748 
DunCAN, M. y Culver, K. B. (2020). Technologies, ethics and journalism's relationship with the public. Media and Communication, 8(3), 101-111. https://doi.org/10.17645/mac.v8i3.3039

EKSTRÖM, M. y WESTLUND, O. (2019). The Dislocation of News Journalism: a Conceptual Framework for the Study of Epistemologies of Digital Journalism. Media and Communication, 7(1), 259-270. http://dx.doi.org/10.17645/mac.v7i1.1763

EKSTRÖM, M.; LEWIS, S. C. y WESTLUND, O. (2020). Epistemologies of digital journalism and the study of misinformation. New Media \& Society, 22(2), 205-212. https://doi.org/10.1177/1461444819856914

FERGUSON, D. A. y GREER, C. F. (2019). Assessing the Diffusion of Drones in Local Television News. Electronic News, 13(1), 23-33. https: / / doi.org/10.1177/1931243119829430

FORD, H. y HUTCHINSON, J. (2019). Newsbots that mediate journalist and audience relationships. Digital Journalism, 7(8), 1013-1031. https://doi.org/10.1080/21670811.2019.1626752

GALlaRdO-CAMACHO, J. y BREIJO, V. R. (2020). Relationships between law enforcement authorities and drone journalists in Spain. Media and Communication, 8(3), 112-122.

https: / / doi.org/10.17645/mac.v8i3.3097

GraEFE, A. y BoHLKEN, N. (2020). Automated Journalism: A Meta-Analysis of Readers' Perceptions of Human-Written in Comparison to Automated News. Media and Communication, 8(3), 50-59.

https://doi.org/10.17645/mac.v8i3.3019

GuTiÉRrez-CANEDA, B.; PÉREZ-SEIJO, S. y LóPEZ-GARcíA, X. (2020). Las secciones y Apps de RV y vídeos 360 a examen. Estudio de caso de siete medios con impacto en Europa. Revista Latina de Comunicación Social, (75), 149-167. https: / / doi.org/10.4185/RLCS-2020-1420

HAMILTON, J. F. (2020). Drone journalism as visual aggregation: Toward a critical history. Media and Communication, 8(3), 64-74. http: / /dx.doi.org/10.17645/mac.v8i3.3117

HASSAN, R. (2020). Digitality, virtual reality and the «empathy machine». Digital Journalism, 8(2), 195-212. https: //doi.org/10.1080/21670811.2018.1517604?journalCode $=$ rdij 20

JONES, B. y JONES, R. (2019). Public service chatbots: Automating conversation with BBC News. Digital Journalism, 7(8), 1032-1053. https://doi.org/10.1080/21670811.2019.1609371

KANG, S.; O'BRIEN, E.; VillarReAL, A.; LEE, W. y MAHOOD, C. (2019). Immersive Journalism and Telepresence: Does virtual reality news use affect news 
credibility? Digital Journalism, 7(2), 294-313.

https://doi.org/10.1080/21670811.2018.1504624

KIM, B. y Yoon, Y. (2019). Journalism Model Based on Blockchain with Sharing Space. Symmetry, 11(1), 1-17. https://doi.org/10.3390/sym11010019

KUKKAKORPI, M. y PANTTI, M. (2020). A Sense of Place: VR Journalism and Emotional Engagement. Journalism Practice, 1-18.

https://doi.org/10.1080/17512786.2020.1799237

KÜMPEL, A. S. (2020). The Matthew Effect in social media news use: Assessing inequalities in news exposure and news engagement on social network sites (SNS). Journalism. https://doi.org/10.1177/1464884920915374

KUNERT, J. (2020). Automation in sports reporting: Strategies of data providers, software providers, and media outlets. Media and Communication, 8(3), 5-15. https://doi.org/10.17645/mac.v8i3.2996

KunERT, J. y THURMAN, N. (2019). The form of content personalisation at mainstream, transatlantic news outlets: 2010-2016. Journalism Practice, 13(7), 759-780.

https: / / doi.org/10.1080/17512786.2019.1567271

LEMELSHTRICH, L. N. (2018). Robot Journalism: Can Human Journalism Survive? World Scientific.

LEWIS, S. C.; SANDERS, A. K. y CARMODY, C. (2019). Libel by algorithm? Automated journalism and the threat of legal liability. Journalism \& Mass Communication Quarterly, 96(1), 60-81.

https://doi.org/10.1177/1077699018755983

LIU, B. y WEI, L. (2019). Machine Authorship In Situ: Effect of news organization and news genre on news credibility. Digital Journalism, 7(5), 635-657. https://doi.org/10.1080/21670811.2018.1510740

LóPEZ-HidALGo, A. (2016). El periodismo que contará el futuro. Chasqui. Revista Latinoamericana de Comunicación, (131), 239-256. https: / /doi.org/10.16921/chasqui.v0i131.2733

MA, Z. (2019). Effects of immersive stories on prosocial attitudes and willingness to help: testing psychological mechanisms. Media Psychology, 126. https://doi.org/10.1080/15213269.2019.1651655

MAÑAS-VINIEGRA, L.; VELOSO, A. I. y SiERRA-SÁnCHEZ, J. (2020). Immersive content with violence: A research using eye tracking with university students in Spain and Portugal. El Profesional de la Información, 29(1). https: / / doi.org/10.3145/epi.2020.ene.08

MAÑAS, L.; GARCÍA, A. L. y MARTÍN, I. J. (2020). Audience attention and emotion in news filmed with drones: A neuromarketing research. Media and Communication, 8(3), 123-136.

https://doi.org/10.17645/mac.v8i3.3081 
MARTínEZ-NicolÁS, M.; SAPERAS, E. y CARRASCO-CAmPOS, Á. (2019). La investigación sobre comunicación en España en los últimos 25 años (19902014). Objetos de estudio y métodos aplicados en los trabajos publicados en revistas españolas especializadas. Empiria: Revista de metodología de ciencias sociales, (42), 37-69.

https://doi.org/10.5944/empiria.42.2019.23250

MASIP, P. y MiCó-SANZ, J. L. (2010). Investigación sobre cibermedios en España. Trayecto hacia la consolidación. El Profesional de la Información, 19(6), 577-584. https://doi.org/10.3145/epi.2010.nov.03

MuRCIA, F. J. y UfARTE, M. J. (2019). Mapa de riesgos del periodismo hi-tech. Hipertext.net, (18), 47-55.

https: / /doi.org/10.31009/hipertext.net.2019.i18.05

NIELSEN, S. L. y SHEETS, P. (2019). Virtual hype meets reality: Users' perception of immersive journalism. Journalism. https://doi.org/10.1177/1464884919869399

PAíno, A. y RodRíGUEZ, M. I. (2019). Proposal for a new communicative model in immersive journalism. Journalism. https://doi.org/10.1177/1464884919869710

PALMER, L. (2020). «Breaking Free» from the Frame: International Human Rights and the New York Times' 360-Degree Video Journalism. Digital Journalism, 8(3), 386-403.

https: / / doi.org/10.1080/21670811.2019.1709982

PAVLIK, J. V. (2020). Drones, augmented reality and virtual reality journalism: Mapping their role in immersive news content. Media and Communication, 8(3), 137-146. https: / /doi.org/10.17645/mac.v8i3.3031

PÉREZ-Luque, M. J. y FERnÁNDEZ-ARRoyo, N. (1995). La integración de la tecnología multimedia y de las redes interactivas: revolución de los sistemas informáticos. Situación, Banco Bilbao-Vizcaya, 4, 105-145.

RAmírez-Montoya, M. S. y GARcíA-PEÑAlVo, F. J. (2018). Co-creación e innovación abierta: Revisión sistemática de literatura. Comunicar, 26(54), 9-18. https: / / doi.org/10.3916/C54-2018-01

RoDRíGUEZ-DE-LAS-HERAS, A. (1991). Navegar por la información. Fundesco. RoJAS, J. (2019). Automated sports coverages. Case study of bot released by The Washington Post during Río 2016 and Pyeongchang 2018 Olympics. Revista Latina de Comunicación Social, (74), 1729-1747.

https://doi.org/10.4185/RLCS-2019-1407en

RoJAS, J. L. y TOURAL, C. (2019). Automated sports journalism. The AnaFut case study, the bot developed by El Confidencial for writing football match reports. Doxa Comunicación, (29), 235-254.

https: / / doi.org/10.31921/doxacom.n29a12 
SALAVERRÍA, R. (2008). Ciberperiodismo: diez años de prensa digital en España. En FERnÁNDEZ SANZ, J. J. (coord.), Prensa especializada actual: doce calas (pp. 355-383). McGraw-Hill.

SAlaverría, R. (2015). Periodismo en 2014: balance y tendencias. Cuadernos de periodistas, (29), 9-22. https: / / cutt.ly/ygqQksB

SAlAVERría, R. (2019). Periodismo digital: 25 años de investigación. Artículo de revisión. El Profesional de la Información, 28(1). https://doi.org/10.3145/epi.2019.ene.01

SÁNCHEZ LAWS, A. L. y UTNE, T. (2019). Ethics guidelines for immersive journalism. Frontiers in Robotics and AI, 6, 1-13. https://doi.org/10.3389/frobt.2019.00028

SÁNCHEZ, J. S.; ORMAECHEA, S. L. y MAÑAS-VINIEGRA, L. (2020). The training in technology subjects of the future journalist in Spain. Revista Latina de Comunicación Social, (75), 189-206.

https: / / www.doi.org/10.4185/RLCS-2020-1422

SIERRA, J.; LIBERAL, S. y MAÑAS-VINIEGRA, L. (2020). La formación en materias de tecnología del futuro periodista en España. Revista Latina de Comunicación Social, (75), 189-206. https://doi.org/10.4185/RLCS-2020-1422

STEINFELD, N. (2020). To Be there when it Happened: Immersive Journalism, Empathy, and Opinion on Sexual Harassment. Journalism Practice, 14(2), 240-258. https://doi.org/10.1080/17512786.2019.1704842

TANDOC, E. C.; YAO, L. J. y WU, S. (2020). Man vs. Machine? The Impact of Algorithm Authorship on News Credibility. Digital Journalism, 8(4), 548562. https://doi.org/10.1080/21670811.2020.1762102

Toural-Bran, C.; Vizoso, Á.; PÉrez-SEIjo, S.; Rodríguez-CASTro, M. y NEGreirAREY, M. C. (2020). Information Visualization in the Era of Innovative Journalism. Routledge.

Ufarte, M. J.; CAlVo, L. M. y Murcia, F. J. (2020). Las tecnologías hi-tech en los grados en Periodismo. Planes de estudios, formación de los periodistas y propuestas de inserción curricular. adComunica: revista científica de estrategias, tendencias e innovación en comunicación, (20), 43-66. https: / / doi.org/10.6035/2174-0992.2020.20.3

UfARTE, M. J. y MANFREDI, J. L. (2019). Algorithms and bots applied to journalism. The case of Narrativa Inteligencia Artificial: structure, production and informative quality. Doxa Comunicación, (29), 213-233. https://doi.org/10.31921/doxacom.n29a11

USKALI, T.; MANNINEN, V.; IKONEN, P. y HoKKANEN, J. (2020). Diffusion of drone journalism: The case of Finland, 2011-2020. Media and Communication, 8(3), 75-84. https://doi.org/10.17645/mac.v8i3.3075 
VAN DAMme, K.; AlL, A.; De MAREZ, L. y VAN LEUVEn, S. (2019). 360 video journalism: Experimental study on the effect of immersion on news experience and distant suffering. Journalism Studies, 20(14), 2053-2076. https://doi.org/10.1080/1461670X.2018.1561208

VAN DER HAAK, B.; PARKS, M. y CASTELLS, M. (2012). The Future of Journalism: Networked Journalism. International Journal of Communication, (6), 29232938.

VÁZQUEZ-HERRERO, J.; Direito-REbollal, S.; SILVA-RodRíGUEZ, A. y LóPEZ-GARCíA, X. (2020). Journalistic Metamorphosis. Media Transformation in the Digital Age. Springer Nature.

VETTEHEN, P. H.; WiltinK, D.; HUISKAMP, M.; SCHAAP, G. y KETELAAR, P. (2019). Taking the full view: How viewers respond to 360 -degree video news. Computers in Human Behavior, 91, 24-32. https://doi.org/10.1016/j.chb.2018.09.018

WADDELL, T. F. (2019a). Can an algorithm reduce the perceived bias of news? Testing the effect of machine attribution on news readers' evaluations of bias, anthropomorphism, and credibility. Journalism \& Mass Communication Quarterly, 96(1), 82-100. https://doi.org/10.1177/1077699018815891

WADDELL, T. F. (2019b). Attribution Practices for the Man-Machine Marriage: How Perceived Human Intervention, Automation Metaphors, and Byline Location Affect the Perceived Bias and Credibility of Purportedly Automated Content. Journalism Practice, 13(10), 1255-1272. https://doi.org/10.1080/17512786.2019.1585197

WANG, Q. (2020). Differentiation and De-differentiation: The Evolving Power Dynamics Between News Industry and Tech Industry. Journalism \& Mass Communication Quarterly, 1-19. https://doi.org/10.1177/1077699020916809

WITSCHGE, T. y DEUZE, M. (2020). From Suspicion to Wonder in Journalism and Communication Research. Journalism \& Mass Communication Quarterly, 116. https://doi.org/10.1177/1077699020912385

WU, S.; TANDOC, E. C. y SALMON, C. T. (2019a). Journalism reconfigured: Assessing human-machine relations and the autonomous power of automation in news production. Journalism Studies, 20(10), 1440-1457. https://doi.org/10.1080/1461670X.2018.1521299

WU, S.; TANDOC, E. C. y SALMON, C. T. (2019b). When journalism and automation intersect: Assessing the influence of the technological field on contemporary newsrooms. Journalism Practice, 13(10), 1238-1254. https://doi.org/10.1080/17512786.2019.1585198 